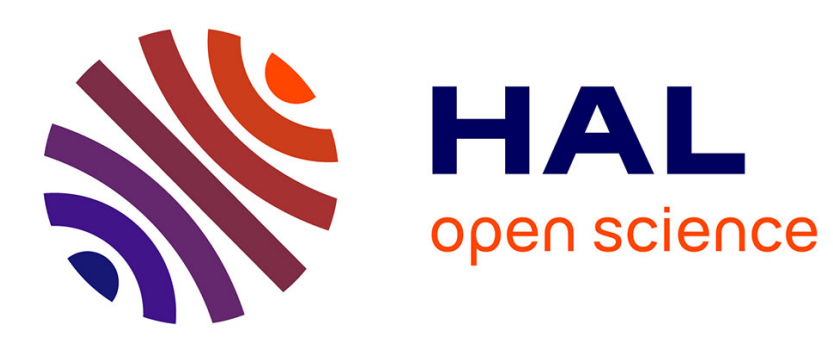

\title{
Sur un nouveau type de modulateur et limiteur hyperfréquences subnanoseconde
}

\author{
B. Boittiaux, Y. Becquelin, J.M. Jendrzejcak, E. Constant
}

\section{To cite this version:}

B. Boittiaux, Y. Becquelin, J.M. Jendrzejcak, E. Constant. Sur un nouveau type de modulateur et limiteur hyperfréquences subnanoseconde. Revue de Physique Appliquée, 1977, 12 (10), pp.1663-1677. 10.1051/rphysap:0197700120100166300 . jpa-00244386

\section{HAL Id: jpa-00244386 https://hal.science/jpa-00244386}

Submitted on 1 Jan 1977

HAL is a multi-disciplinary open access archive for the deposit and dissemination of scientific research documents, whether they are published or not. The documents may come from teaching and research institutions in France or abroad, or from public or private research centers.
L'archive ouverte pluridisciplinaire HAL, est destinée au dépôt et à la diffusion de documents scientifiques de niveau recherche, publiés ou non, émanant des établissements d'enseignement et de recherche français ou étrangers, des laboratoires publics ou privés. 


\title{
SUR UN NOUVEAU TYPE DE MODULATEUR ET LIMITEUR HYPERFRÉQUENCES SUBNANOSECONDE
}

\section{B. BOITTIAUX, Y. BECQUELIN, J. M. JENDRZEJCZAK et E. CONSTANT}

Centre Hyperfréquences et Semiconducteurs $\left({ }^{*}\right)$, Université des Sciences et Techniques de Lille, B.P. 36, 59650 Villeneuve d'Ascq, France

(Reçu le 28 mars 1977, accepté le 17 juin 1977)

\begin{abstract}
Résumé. - Nous proposons une étude théorique et expérimentale d'un nouveau type de composant semiconducteur à variation de mobilité par effet de champ qui devrait permettre la réalisation de modulateurs et de limiteurs hyperfréquences ultra-rapides.

L'étude théorique des différents phénomènes physiques intervenant dans la structure semiconductrice unipolaire utilisée est effectuée analytiquement puis numériquement. Les performances susceptibles d'être obtenues en limiteur ou en modulateur sont calculées. Les premières études expérimentales effectuées sur des échantillons réalisés en silicium permettent alors la vérification des prévisions théoriques.

Enfin, les résultats obtenus sur des premières maquettes non optimalisées montrent l'intérêt que pourraient présenter ces nouveaux dispositifs: temps de commutation inférieur à 100 ps pour le modulateur, limitation pratiquement instantanée de la puissance hyperfréquence pour le limiteur.

Abstract. - In this paper, a theoretical and experimental study of a new microwave device using field effect variation of carrier mobility is presented.

First, the theoretical study of physics mechanisms which occur in the studied unipolar bulk semiconductor is carried out by means of analytical and computer calculations.

Then, experimental results obtained on some $\mathrm{N}^{+} \mathrm{N} \mathrm{N}^{+}$silicon samples are presented and good agreements with theoretical calculations are obtained.

Finally, experimentally switching time of the microwave modulations appears to be well under $100 \mathrm{ps}$; and the power leakage of this type of microwave limiters is practically negligible.
\end{abstract}

1. Introduction. - Dans de nombreux systèmes radars et de télécommunication, il est nécessaire d'effectuer une modulation rapide, analogique ou digitale, de l'amplitude ou de la phase d'une onde hyperfréquence. Les dispositifs utilisés dans ce but sont généralement réalisés à partir de structures semiconductrices dont on peut faire varier dans de grandes proportions la conductivité de la zone active et, en conséquence, la conductance présentée par le composant. Ces variations sont souvent obtenues en injectant ou en produisant des porteurs de charge dans la zone active. Le premier procédé présente un inconvénient essentiel : le temps d'injection des porteurs est limité par les phénomènes de transit dans la structure, tandis que le temps de disparition est lié à leur durée de vie. Il en résulte des temps de commutation généralement élevés dépassant le plus souvent plusieurs nanosecondes. Le second procédé est plus rapide mais il nécessite des puissances de commande très élevées.

(*) E.R.A. au C.N.R.S. $\mathrm{N}^{\circ} 454$.
Dans ces conditions, on peut se demander dans quelle mesure d'autres phénomènes physiques ne pourraient être utilisés. En particulier, il est possible de modifier la mobilité des porteurs libres dans un semiconducteur en faisant varier l'amplitude du champ électrique appliqué de façon à passer du régime de mobilité $(v=\mu E)$ au régime de limitation de vitesse $\left(v=v_{\mathrm{s}}\right.$ : mobilité différentielle $\left.\mu_{\mathrm{d}}=0\right)$. Ce changement est très rapide puisqu'il est associé à des transitions intravallées et intervallées dont les effets se manifestent en des temps inférieurs à quelques picosecondes. Quelques publications ont porté sur l'utilisation de ce procédé $[1,2]$ mais elles ne concernent que le $\mathrm{GaAs}_{1-x} \mathrm{P}_{x}$ et le fonctionnement en modulateur. Nous présentons ici une étude plus générale des possibilités de modulateurs et de limiteurs fondés sur ce principe et réalisés à partir de semiconducteurs usuels ( $\mathrm{Si}, \mathrm{GaAs}, \mathrm{Ge}$ ) mais aussi de composés ternaires tels que le $\mathrm{Ga}_{x} \mathrm{In}_{1-x} \mathrm{Sb}$.

Le principe de ces modulateurs ou limiteurs est très simple. La structure semiconductrice utilisée est par exemple placée en série entre l'émetteur et le 
récepteur. En l'absence de polarisation continue ou à faible niveau incident, l'impédance présentée par le composant doit être faible de façon que toute la puissance hyperfréquence soit transmise; par contre, lorsque la polarisation continue est appliquée ou à fort niveau hyperfréquence, l'impédance doit être alors importante pour que la puissance transmise devienne négligeable.

Dans ce but, nous nous proposons d'étudier et d'utiliser les propriétés d'une structure semiconductrice unipolaire; l'objectif fixé est de créer, par l'application d'un champ électrique continu (modulateur) ou hyperfréquence (limiteur), une variation maximale de la mobilité des porteurs dans la zone active, ou en d'autres termes, sous l'effet d'une tension continue ou alternative une modification maximale de la conductance présentée par l'échantillon. Il est bien entendu que ces variations devraient être obtenues pour des puissances de commande minimales.

Dans un premier temps, nous envisageons une théorie très simplifiée du fonctionnement de la structure semiconductrice et progressivement, nous approfondissons notre étude et mettons en évidence les différents phénomènes physiques intervenant dans le dispositif, qualitativement sous forme analytique puis quantitativement sous forme numérique.

Dans la seconde partie, nous donnons et comparons avec la théorie les premiers résultats expérimentaux obtenus sur des structures réalisées par la Société L.T.T. (1) et par notre laboratoire.

Nous envisageons ensuite plusieurs applications du contraste de conductance obtenu dans la gamme des hyperfréquences et nous nous efforçons d'évaluer d'une façon aussi réaliste que possible, les performances prévisibles de modulateurs et de limiteurs rapides réalisés à partir des structures semiconductrices unipolaires. Quelques résultats préliminaires obtenus sur des maquettes très simples viennent étayer ces prévisions.

1. Étude des propriétés statiques et hyperfréquences d'une structure semiconductrice unipolaire soumise à une tension continue ou hyperfréquence importante. - La structure semiconductrice étudiée est représentée figure 1, elle est de type $\mathrm{N}$ et ses propriétés de symétrie permettent une analyse unidimensionnelle. Malgré la simplicité du composant envisagé, l'étude des phénomènes physiques va devenir rapidement complexe lorsque la structure semiconductrice sera soumise à une tension continue ou hyperfréquence importante. En effet, si la vitesse d'entraînement des porteurs va rapidement se saturer lorsque le champ électrique va augmenter, l'injection de porteurs excédentaires dans la zone active du semiconducteur va simultanément créer une charge d'espace et en conséquence une non uniformité du

(1) Lignes Télégraphiques et Téléphoniques 78700 Conflans Ste Honorine

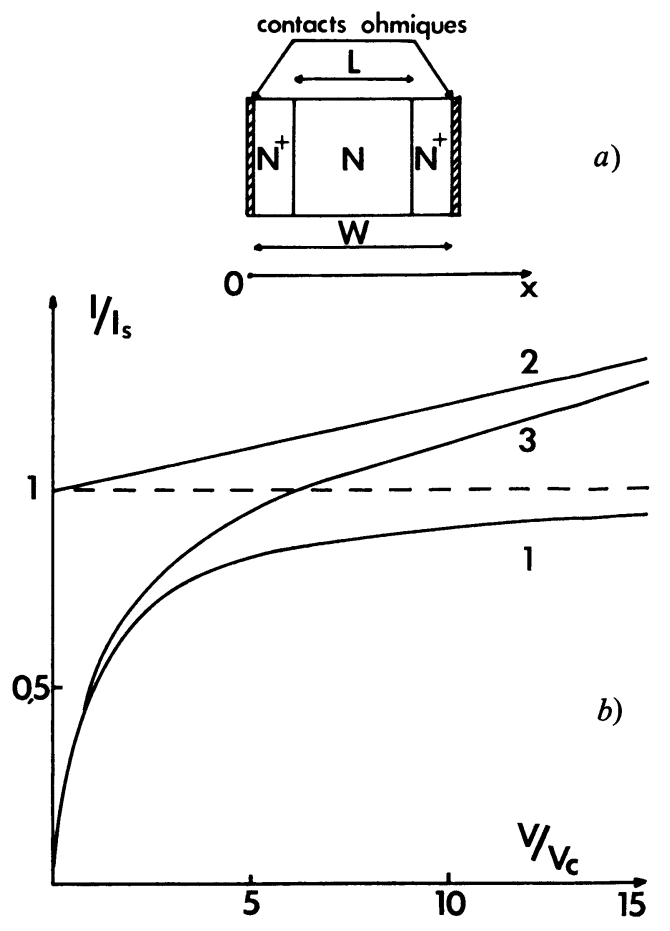

Fig. 1. - A) Structure semiconductrice $\mathrm{N}^{+} \mathrm{N} \mathrm{N}^{+}$; B) Caractéristiques courant-tension normalisées; 1 , effet de la limitation de vitesse : relation (3); 2 , effet de charge d'espace : relation (9); 3 , loi approchée : relation (11).

[A) $\mathrm{N}^{+} \mathrm{N} \mathrm{N}^{+}$Semiconductor structure; B) Normalized $I(V)$ characteristics; 1, with saturated velocity effect (equation 3); 2 , with space charge effect (equation 9); 3 , approached relation (equation 11).]

champ électrique. Enfin, sous l'action du champ hyperfréquence, des porteurs vont être injectés périodiquement dans la structure et en se propageant vont créer des effets dépendant directement de leur temps de transit. Afin de mettre bien en évidence la contribution de ces différents phénomènes sur l'admittance présentée par l'échantillon, nous allons successivement étudier les effets liés à la saturation de la vitesse, puis à la charge d'espace et enfin à l'injection et au temps de transit des porteurs. Nous adopterons ainsi progressivement des hypothèses de moins en moins restrictives dans cette étude qui, commencée de façon analytique, se terminera de façon numérique lorsque la difficulté des calculs deviendra trop importante.

1.1. Étude analytiQue. - 1.1.1. Effet de la saturation de la vitesse. Pour bien mettre en évidence l'évolution de la conductance en fonction de la tension appliquée, l'hypothèse la plus simple est d'admettre que le champ électrique est constant quelle que soit l'amplitude de la tension dans toute la longueur de la zone active du semiconducteur et, qu'il est nul dans les zones $\mathrm{N}^{+}$(supposées dans un premier temps infiniment dopées avec des transitions $\mathbf{N}^{+} \mathbf{N}$ parfaitement abruptes) (cf. Fig. 1).

Dans ces conditions, le nombre de porteurs mobiles par unité de volume est égal à $N_{\mathrm{D}}$ et le courant tra- 
versant l'échantillon de surface $S$ soumis à une tension $V$ est donné par la relation :

$$
I=q N_{\mathrm{D}} S v(V / L)
$$

Le rapport des valeurs maximale et minimale de la conductance présentée par la structure ne dépend ainsi que de la caractéristique $v=\mathrm{f}(E)$ : vitesse des porteurs libres fonction du champ électrique du semiconducteur utilisé. Dans cette étude simplifiée, nous supposerons que :

$$
v(E)=\frac{\mu_{\mathrm{o}} E}{1+\left|E / E_{\mathrm{c}}\right|}
$$

$\mu_{\mathrm{o}}$ : mobilité à champ électrique faible

$E_{\mathrm{c}}$ : champ critique tel que $v_{\mathrm{s}}=\mu_{\mathrm{o}} E_{\mathrm{c}}$

$v_{\mathrm{s}}:$ vitesse limite des porteurs.

Cette expression est assez bien vérifiée dans le cas du germanium et du silicium, mais elle constitue évidemment une approximation beaucoup plus importante dans le cas des semiconducteurs à mobilité différentielle négative tel que l'arséniure de gallium.

A partir des expressions (1) et (2) on déduit facilement la relation courant-tension :

$$
I=I_{\mathrm{s}} \frac{V / V_{\mathrm{c}}}{1+\left|V / V_{\mathrm{c}}\right|}
$$

avec $I_{\mathrm{s}}=S q N_{\mathrm{D}} v_{\mathrm{s}}$ et $V_{\mathrm{c}}=E_{\mathrm{c}} L$

Dans un fonctionnement en modulateur, la tension appliquée est une tension continue; le rapport conductance différentielle à champ faible $g_{\mathrm{o}}$ sur la conductance différentielle à champ fort $g_{v}\left({ }^{2}\right)$ s'obtient immédiatement de la relation (3) :

$$
\frac{g_{\mathrm{o}}}{g_{\mathrm{v}}}=\left(1+\left|V / V_{\mathrm{c}}\right|\right)^{2}
$$

En limiteur, la tension appliquée est de la forme : $V(t)=V_{1} \cos 2 \pi F t$ et l'analyse harmonique de $I(t)$ permet de trouver à la fréquence d'étude $F$ le rapport des conductances $g_{\mathrm{o}} / \tilde{g}_{\mathrm{v}}$. Dans le cas où $V_{1} \gg V_{\mathrm{c}}$ l'évolution de $g_{\mathrm{o}} / \tilde{g}_{\mathrm{v}}$ est reliée à l'amplitude du signal par la relation approchée :

$$
\frac{g_{\mathrm{o}}}{\tilde{g}_{\mathrm{v}}} \simeq 1+\frac{\pi}{4} \frac{V_{1}}{V_{\mathrm{c}}}
$$

Les relations (4) et (5) nous donnent la valeur maximale du rapport de conductance qui sera obtenue lorsque la tension appliquée au semiconducteur sera voisine de la tension d'avalanche. La puissance

(2) En parallèle sur cette conductance, il existera toujours une capacité liée au courant de déplacement.

REVUE DE PHYSIQUE APPLIQUÉE. - T. 12, No 10, OCTOBRE 1977 nécessaire pour obtenir cette variation est alors exprimée par les relations suivantes :

$$
\begin{aligned}
& P_{\mathrm{c}}=S N_{\mathrm{D}} L q v_{\mathrm{s}} E_{\mathrm{MAX}} \quad \text { (modulateur) } \\
& \tilde{P}_{\mathrm{c}}=\frac{2}{\pi} S N_{\mathrm{D}} L q v_{\mathrm{s}} E_{\mathrm{MAX}} \quad \text { (limiteur) }
\end{aligned}
$$

Quelques valeurs numériques calculées à partir des relations (4) à (7) sont reportées tableau I pour les semiconducteurs les plus usuels. A la lumière de ces résultats, les remarques suivantes peuvent être effectuées :

- d'une part, les variations de conductance susceptibles d'être obtenues à partir d'une tension continue sont beaucoup plus importantes que celles provoquées par une tension hyperfréquence,

- d'autre part, les semiconducteurs tels que $\mathrm{GaAs}$

\begin{tabular}{|c|c|c|c|c|c|}
\hline \multicolumn{2}{|c|}{$\begin{array}{c}\text { SEMI- } \\
\text { CONDUCTEURS }\end{array}$} & $\mathrm{Ge}$ & $\mathrm{S}$ & GaAs & $\begin{array}{c}{[5] \quad[6]} \\
\mathrm{Ga}_{x} \operatorname{In}_{1-x} \mathrm{Sb} \\
x=0,9\end{array}$ \\
\hline $\begin{array}{l}\mathrm{C} \\
\mathrm{O}\end{array}$ & & & & & \\
\hline $\begin{array}{l}\mathrm{N} \\
\mathrm{S}\end{array}$ & $\begin{array}{c}v_{\mathrm{s}} \times 10^{-7} \\
(\mathrm{~cm} / \mathrm{s})\end{array}$ & 0,7 & 1,0 & 1,0 & 0,8 \\
\hline $\begin{array}{l}\mathrm{T} \\
\mathrm{A}\end{array}$ & $\begin{array}{c}\mu_{\mathrm{o}} \times 10^{-3} \\
\left(\mathrm{~cm}^{2} \mathrm{~s}^{-1} \mathrm{v}^{-1}\right)\end{array}$ & 3,5 & 1,45 & 5,0 & 14,0 \\
\hline $\begin{array}{l}\mathrm{N} \\
\mathrm{T} \\
\mathrm{E} \\
\mathrm{S}\end{array}$ & $\begin{array}{c}E_{\mathrm{c}} \\
(\mathrm{kV} / \mathrm{cm})\end{array}$ & 2,0 & 6,9 & 2,0 & 0,57 \\
\hline $\begin{array}{l}\mathrm{M} \\
\mathrm{O} \\
\mathrm{D}\end{array}$ & $\begin{array}{c}E_{\max } \times 10^{-2} \\
(\mathrm{kV} / \mathrm{cm})\end{array}$ & 0,8 & 2,0 & 2,0 & 1,0 \\
\hline $\begin{array}{l}\mathrm{U} \\
\mathrm{L}\end{array}$ & $\frac{g_{v}}{g_{v}} \times 10^{-3}$ & 1,68 & 0,89 & 10,2 & 31 \\
\hline $\begin{array}{l}\mathrm{A} \\
\mathrm{T} \\
\mathrm{E} \\
\mathrm{U} \\
\mathrm{R} \\
\end{array}$ & $\begin{array}{l}\frac{P_{\mathrm{c}}}{S N_{\mathrm{D}} L} \times 10^{+7} \\
\cdot(\mathrm{W})\end{array}$ & 0,87 & 3,1 & 3,2 & 1,3 \\
\hline L & $\begin{array}{c}\tilde{E}_{1 \max } \times 10^{-2} \\
(\mathrm{kV} / \mathrm{cm})\end{array}$ & 1,2 & 3,0 & 3,0 & 1,5 \\
\hline $\begin{array}{l}\text { M } \\
\text { I }\end{array}$ & $\cdot \frac{g_{0}}{\tilde{\tilde{g}_{v}}} \times 10^{-2}$ & 0,47 & 0,34 & 1,18 & 2,07 \\
\hline $\begin{array}{l}T \\
\mathrm{E} \\
\mathrm{U} \\
\mathrm{R}\end{array}$ & $\begin{array}{c}\frac{P_{\mathrm{c}}}{S N_{\mathrm{D}} L} \times 10^{+7} \\
(\mathrm{~W})\end{array}$ & 0,86 & 3,1 & 3,1 & 1,2 \\
\hline
\end{tabular}
et $\mathrm{Ga}$ In $\mathrm{Sb}$ présentant une mobilité élevée et un champ critique faible s'avèrent les plus intéressants à utiliser pour ce type d'application.

1.1.2. Effets de la charge d'espace. - L'étude précédente est très simplifiée car elle ne tient aucun compte

TABLEAU I 
de la charge d'espace. En effet, à la cathode, le champ électrique passe d'une valeur nulle dans la zone $\mathrm{N}^{+}$ à une valeur élevée dans la zone $\mathrm{N}$ et d'après la loi de Poisson, la concentration en porteurs libres doit obligatoirement être supérieure à $N_{\mathrm{D}}$. Des charges électriques excédentaires vont donc apparaître dans la zone active et le champ électrique dans la structure ne sera plus constant, nous nous proposerons d'étudier l'influence de ces phénomènes sur les valeurs de la conductance présentée par l'échantillon.

Montrons tout d'abord que contrairement à ce que prévoit la relation (3) il est possible d'avoir un courant $I$ supérieur à $I_{\mathrm{s}}$; il suffit que le champ électrique dans la structure soit suffisamment élevé afin que la vitesse limite soit atteinte dans toute la zone active. On a alors :

$$
I=S q n v_{\mathrm{s}}
$$

$n:$ concentration en porteurs libres.

Dans le cas où le courant est supérieur à $I_{\mathrm{s}}, n$ est supérieur à $N_{\mathrm{D}}$ et égal à une constante dans toute la zone active du semiconducteur. D'après la loi de Poisson le champ électrique croît alors linéairement de la cathode à l'anode et une intégration simple montre que si $V \gg V_{\mathrm{c}}$ :

$$
I-I_{\mathrm{s}}=g_{\mathrm{c}} V
$$

où $g_{\mathrm{c}}$ : conductance différentielle de charge d'espace s'exprime par :

$$
g_{\mathrm{c}}=2 \varepsilon S v_{\mathrm{s}} / L^{2}
$$

( $\varepsilon$ est la permittivité du semiconducteur utilisé).

La relation (9) n'est évidemment valable que pour des tensions très élevées et constitue en réalité la limite asymptotique de la caractéristique $I(V)$. Une expression approchée plus réaliste peut être obtenue à partir des relations (3) et (9) :

$$
I \neq \neq I_{\mathrm{s}} \frac{V / V_{\mathrm{c}}}{1+V / V_{\mathrm{c}}}+g_{\mathrm{c}} V
$$

Les valeurs de la conductance dynamique présentée par l'échantillon se déduisent directement de la pente de la caractéristique $I(V)$ (Fig. 1) et seront toujours supérieures à la valeur de $g_{\mathrm{c}}$. La valeur relative maximale de la conductance est donc donnée par :

$$
\frac{g_{\mathrm{o}}}{g_{\mathrm{c}}}=\frac{q \mu_{\mathrm{o}} N_{\mathrm{D}} L}{2 \varepsilon v_{\mathrm{s}}}=\frac{t_{\mathrm{T}}}{2 \rho \varepsilon}=\frac{t_{\mathrm{T}}}{2 \tau}
$$

$\rho$ : résistivité du semiconducteur considéré

$t_{\mathrm{T}}=L / v_{\mathrm{s}}=$ temps de transit des porteurs dans la zone active

$\tau:$ temps de relaxation du semiconducteur.

Par ailleurs, les variations de la conductance sous l'effet d'une polarisation continue ou hyperfréquence forte s'obtiennent respectivement à partir des nouvelles expressions approchées suivantes :

$$
\begin{aligned}
& \frac{g_{\mathrm{o}}}{g} \neq \neq\left[\frac{1}{\left(1+V / V_{\mathrm{c}}\right)^{2}}+\frac{2 \varepsilon v_{\mathrm{s}}}{q \mu_{\mathrm{o}} N_{\mathrm{D}} L}\right]^{-1} \\
& \frac{g_{\mathrm{o}}}{\tilde{g}} \neq \neq\left[\frac{4}{\pi} \frac{V_{\mathrm{c}}}{\tilde{V_{1}}}+\frac{2 \varepsilon v_{\mathrm{s}}}{q \mu_{\mathrm{o}} N_{\mathrm{D}} L}\right]^{-1}
\end{aligned}
$$

On remarque que l'effet d'injection est d'autant plus faible que le produit $N_{\mathrm{D}} L$ est grand, cependant les puissances de commandes (relations (6) et (7)) sont directement proportionnelles à ce terme et une solution optimale dépendant des performances recherchées devra être trouvée. A titre d'exemple, dans le cas où la contribution des effets de la saturation de la vitesse a la même importance que les effets d'injection $\left(g_{\mathrm{v}} \simeq g_{\mathrm{c}}\right)$, nous donnons dans le tableau II la valeur de $N_{\mathrm{D}} L$ le champ électrique et la puissance de

\begin{tabular}{|c|c|c|c|c|c|}
\hline \multicolumn{2}{|c|}{$\begin{array}{c}\text { SEMI- } \\
\text { CONDUCTEURS }\end{array}$} & \multirow{2}{*}{\multicolumn{2}{|c|}{\begin{tabular}{|ll} 
Ge Si \\
16,311,
\end{tabular}}} & \multirow{2}{*}{$\frac{\mathrm{GaAs}}{712}$} & \multirow{3}{*}{$\frac{\mathrm{Ga}_{x} \mathrm{In}_{1-x} \mathrm{Sb}}{15}$} \\
\hline & $\varepsilon_{\mathrm{r}}$ & & & & \\
\hline $\begin{array}{ll}M & \text { a } \\
\mathrm{O} & \|\end{array}$ & & & & & \\
\hline $\begin{array}{l}\mathrm{D} \\
\mathrm{U}\end{array}$ & $\underset{(\mathrm{kV} / \mathrm{cm})}{E}$ & 26,3 & 90,7 & 26,3 & 7,5 \\
\hline $\begin{array}{l}\mathrm{L} \\
\mathrm{A}\end{array}$ & $\begin{array}{c}N_{\mathrm{D}} L \times 10^{-12} \\
\left(\mathrm{~cm}^{-2}\right)\end{array}$ & 7,2 & 18 & 5,3 & 1,9 \\
\hline $\begin{array}{ll}\mathrm{T} & \\
\mathrm{E} & \| \\
\mathrm{U} & \infty \\
\mathrm{R} & \infty \\
\end{array}$ & $\begin{array}{c}P / S \times 10^{-5} \\
\left.(\mathrm{~W} \mathrm{~cm})^{-2}\right)\end{array}$ & 2,3 & 28 & 2,4 & 0,19 \\
\hline $\begin{array}{l}\mathrm{L} \\
\mathrm{I} \\
\mathrm{M}\end{array}$ & $\begin{array}{c}\tilde{E} \\
(\mathrm{kV} / \mathrm{cm})\end{array}$ & 76,4 & 264 & 76,4 & 21,7 \\
\hline $\begin{array}{ll}I & 0 \\
T & 0\end{array}$ & $\begin{array}{c}N_{\mathrm{D}} L \times 10^{-11} \\
\left(\mathrm{~cm}^{-2}\right)\end{array}$ & 10,8 & 26,9 & 8 & 2,8 \\
\hline $\begin{array}{l}\mathrm{E} \\
\mathrm{U}\end{array}$ & $\begin{array}{c}\tilde{P} / S \times 10^{-5} \\
\left(\mathrm{~W} / \mathrm{cm}^{2}\right)\end{array}$ & 1,5 & 18,6 & 1,6 & 0,13 \\
\hline 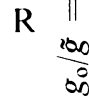 & & & & & \\
\hline
\end{tabular}
commande par unité de surface pour obtenir $g_{\mathrm{o}} / g=$ 100 en modulateur et $g_{\mathrm{o}} / \tilde{g}=15$ en limiteur.

\section{TABLEAU II}

Valeurs calculées lorsque des effets liés à la limitation de la vitesse des porteurs ont la même contribution que les effets liés à la charge d'espace.

1.1.3. Effet d'injection hyperfréquence. - Toutes les propriétés précédentes sont valables à des fréquences suffisamment faibles pour que les temps de 
transit soient négligeables, c'est-à-dire pour des angles de transit $\theta=2 \pi F L / v \ll 1$. Nous allons maintenant nous placer dans le cas où les angles de transit deviennent importants. L'expression de l'admittance en régime linéaire, c'est-à-dire pour un fonctionnement en modulateur, est très classique $[3,4]$ et elle peut se mettre, moyennant quelques simplifications que nous allons préciser, sous la forme très générale :

$$
Y(\omega)=Y_{\text {nat }}\left[1-I^{*} \frac{\mathrm{e}^{\gamma L}-1}{\gamma L}\right]^{-1}=g+j b
$$

Dans cette expression :

$Y_{\text {nat }}=\frac{S\left(\sigma_{\mathrm{d}}+j \omega \varepsilon\right)}{L} \quad\left(\operatorname{avec} \sigma_{\mathrm{d}}=q n \mu_{\mathrm{d}} \neq \neq g_{\mathrm{v}} L / S\right)$

représente, compte tenu du courant de déplacement, l'admittance présentée par l'échantillon semiconducteur lorsque les effets d'injection sont négligeables. La grandeur :

$$
I^{*}=\frac{\sigma_{i}-\sigma_{d}}{\sigma_{i}+j \omega \varepsilon}
$$

caractérise les propriétés de la zone injectante. Elle dépend de $\sigma_{i}$, la conductivité d'injection définie à partir des relations existant à l'originé (c'est-à-dire à la cathode) entre le champ électrique et le courant hyperfréquence

$$
\tilde{j}=\tilde{e}(0) \quad\left(\sigma_{\mathrm{i}}+j \omega \varepsilon\right)
$$

L'origine est généralement choisie de façon que le champ continu y soit nul et en conséquence la conductivité d'injection peut en première approximation être prise égale à l'infini. Enfin la constante de propagation de la charge d'espace est donnée par :

$$
\gamma=\frac{v}{2 D}-\frac{v}{2 D} \sqrt{1+\frac{4 D}{v^{2}} \frac{\left(\sigma_{\mathrm{d}}+j \omega \varepsilon\right)}{\varepsilon}}
$$

où $D$ est le coefficient de diffusion des porteurs dans le semiconducteur et il est important de préciser que la relation (15) est obtenue en supposant $\gamma$ indépendant de $x$ dans la zone active du semiconducteur.

Pour des champs électriques importants, les porteurs se déplacent à une vitesse voisine de la vitesse limite $\left(\sigma_{d} \rightarrow O\right)$ et le coefficient de diffusion diminue. En conséquence, la constante de propagation peut alors s'obtenir avec une bonne précision en utilisant un développement au second ordre soit :

$$
\gamma=-\frac{\sigma_{\mathrm{d}}}{\varepsilon v}+\frac{D}{v^{3} \varepsilon^{2}}\left(\sigma_{\mathrm{d}}^{2}-\omega^{2} \varepsilon^{2}\right)-j \frac{\omega}{v}
$$

Les relations (15) et (17) permettent le calcul de la conductance présentée par l'échantillon et l'interpré- tation des résultats peut se faire commodément en introduisant une fonction $f(\theta)$ définie par la relation :

$g=g_{\mathrm{c}} f(\theta)$

Les variations de $f(\theta)$ sont représentées figure 2 pour différentes valeurs de la conductivité différentielle $\sigma_{\mathrm{d}}$ et du coefficient de diffusion. On remarque que dans le cas où ces deux paramètres tendent vers zéro la fonction $f(\theta)$ s'annule pour les valeurs de l'angle de transit multiples de $2 \pi$ et les effets de la charge d'espace peuvent être ainsi éliminés. Lorsque le champ électrique est plus faible $\left(v<v_{\mathrm{s}}, \sigma_{\mathrm{d}} \neq \neq \mathrm{O}\right)$ ou lorsque le phénomène de diffusion ne peut pas être négligé, la constante de propagation présente une partie réelle négative; les effets liés au temps de transit sont alors atténués (Fig. 2, courbes 2 et 3 ) et il n'est plus possible d'obtenir des conductances $g$ notablement inférieures à $g_{\mathrm{c}}$. C'est en particulier le cas aux fréquences élevées lorsque le coefficient de diffusion n'est pas négligeable (Fig. 2, courbe 3).

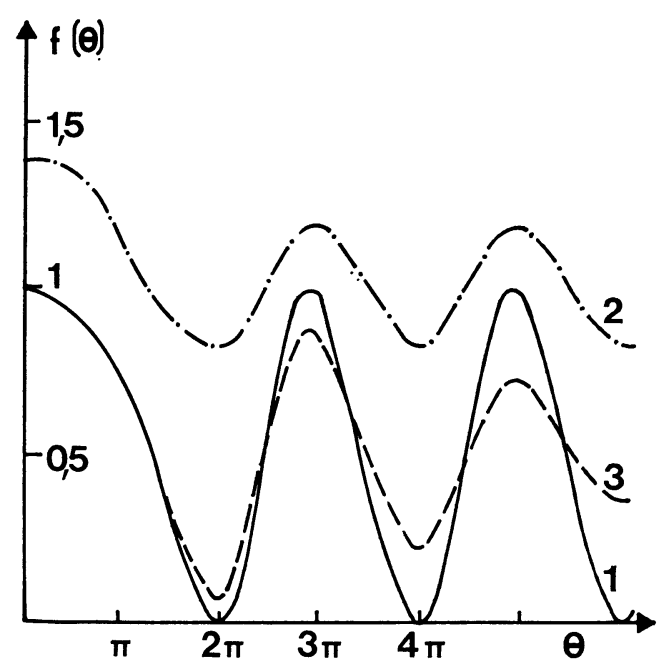

FIG. 2. - Influence de l'injection hyperfréquence sur le facteur de transport $f(\theta)$. 1. $\sigma_{\mathrm{d}}=0 ; D=0 ; 2 . \sigma_{\mathrm{d}} \neq 0\left(g_{\mathrm{v}}=g_{\mathrm{c}}\right) ; D=0$; 3. $\sigma_{\mathrm{d}}=0 ; D=35 \mathrm{~cm}^{2} \cdot \mathrm{s}^{-1}$.

[Microwave injection contribution on the drift factor $f(\theta)$. 1. $\sigma_{\mathrm{d}}=0 ; D=0 ; 2$. $\sigma_{\mathrm{d}} \neq 0\left(g_{\mathrm{v}}=g_{\mathrm{c}}\right) ; D=0 ; 3 . \sigma_{\mathrm{d}}=0$; $\left.D=35 \mathrm{~cm}^{2} \cdot \mathrm{s}^{-1}.\right]$

En conclusion, l'étude analytique a permis :

- de mettre en évidence les rôles respectifs de la saturation de la vitesse des porteurs, de la charge d'espace et du temps de transit,

- de donner un ordre de grandeur de leur contribution.

Les résultats qualitatifs obtenus montrent que pour obtenir un contraste de conductance maximum sans 
que les puissances de commande soient trop importantes, il faut choisir un semiconducteur de vitesse limite faible et de grande mobilité (relations (6) et (7)). Dans ce contexte, le GaAs et le GaInSb [5] paraissent les matériaux les plus intéressants. Par ailleurs, nous avons vu que les effets d'injection pouvaient être diminués :

- soit en choisissant des structures caractérisées par des produits $N_{\mathrm{D}} L$ importants,

- soit dans le domaine des hyperfréquences, en réalisant (pour un fonctionnement en modulateur) des structures caractérisées par des angles de transit voisins de $2 \pi$.

La théorie analytique effectuée est cependant très simplifiée; elle ne nous a pas permis de tenir compte :

- des transitions $\mathrm{N}^{+} \mathbf{N}$ pratiquement toujours nécessaires pour réaliser un bon contact ohmique métal semiconducteur,

- de la non uniformité du champ électrique dans l'évaluation des effets du temps de transit et dans le fonctionnement en limiteur,

- de la variation du coefficient de diffusion avec l'amplitude du champ électrique.

Par ailleurs, pour ce type de composants rapides, l'étude du régime transitoire est évidemment essentielle; or elle ne peut que difficilement se traiter de façon analytique [7]. En conséquence, nous avons développé une méthode de résolution numérique permettant de décrire le comportement du composant soumis à une tension continue ou hyperfréquence d'amplitude quelconque ainsi que son régime transitoire.

1. 2. ÉtUde NUMÉriQue. - Nous considérons ici un modèle unidimensionnel et nous supposons que la vitesse et le coefficient de diffusion des porteurs sont des fonctions instantanées du champ électrique. Dans ces conditions, l'expression de la densité de courant à un instant $t$ s'écrit :

$$
J(t)=J_{\mathrm{c}}(x, t)+J_{\mathrm{d}}(x, t)+J_{\mathrm{dep}}(x, t)
$$

avec :

$$
\begin{aligned}
J_{\mathrm{c}}(x, t) & =-q n(x, t) v[E(x, t)] \\
J_{\mathrm{d}}(x, t) & =q D[E(x, t)] \frac{\partial}{\partial x} n(x, t) \\
J_{\text {dep }}(x, t) & =\varepsilon \frac{\partial E(x, t)}{\partial t}
\end{aligned}
$$

En combinant avec l'équation de Poisson :

$$
\varepsilon \frac{\partial E(x, t)}{\partial x} \doteq-q\left[n(x, t)-N_{\mathrm{d}}(x)\right]
$$

on obtient une équation non linéaire aux dérivées partielles dont la seule inconnue est $E(x, t)$. Pour la résoudre, [8] il faut connaître les expressions des fonctions $v[E(x, t)]$ et $D[E(x, t)]$ et passer aux différences finies en considérant un pas $\Delta x$ sur l'espace et un pas $\Delta t$ sur le temps. La relation obtenue se simplifie considérablement en posant :

$v[E(x, t)]=v[E(x, t-\Delta t)]$ et $D[E(x, t)]=D[E(x, t-\Delta t)]$

Cette hypothèse permet un gain important de temps calcul [8] par rapport aux méthodes itératives et donne de très bons résultats à condition de respecter les conditions de stabilité [8]: $\Delta t<\min \left(\frac{\Delta x^{2}}{2 D} ; \frac{2 D}{v^{2}}\right)$ et $\Delta t<\rho(x)_{\min } \varepsilon$. En se donnant les conditions initiales, c'est-à-dire $E(\mathrm{O}, t)$ et $E(W, t)$ le système d'équations se résoud par la méthode du double balayage de Choleski [9]. Pour un composant déterminé, (lois $v(E)$ et $D(E)$, caractéristiques du semiconducteur utilisé, dimensions géométriques, profil de concentration en impuretés) le programme numérique permet :

- d'obtenir à chaque instant $t_{\mathrm{i}}$

- la carte du champ électrique dans l'échantillon,

- la répartition des porteurs libres,

- la vitesse des porteurs,

- la tension apparaissant aux bornes $V\left(t_{\mathrm{i}}\right)$,

- de calculer à la fréquence fondamentale et aux différents harmoniques,

- l'impédance de l'échantillon $Z\left(\omega_{\mathrm{k}}\right)$,

- la puissance absorbée par l'échantillon $P\left(\omega_{k}\right)$.

En pratique, le courant traversant la structure semiconductrice est imposé à priori. En régime petit signal on prend :

$$
I(t)=I_{\mathrm{o}}+I_{1} \sin 2 \pi F t \text { avec } I_{\mathrm{o}} \gg I_{1}
$$

L'étude grand signal est réalisée dans le cas où :

$I(t)=I_{1} \sin 2 \pi F t$

La connaissance de $I(t)$ permet la détermination de $E(t)$ dans les régions fortement dopées $\mathrm{N}^{+}$du semiconducteur et donc l'établissement des conditions aux limites $E(\mathrm{O}, t)$ et $E(W, t)$.

A titre d'exemple, nous donnons les résultats obtenus pour un échantillon de silicium de type $\mathrm{N}$, de résistivité $\rho \simeq 1,6 \Omega \mathrm{cm}$. La transition entre les zones $N^{+}$et $N$ est décrite par la fonction erreur complémentaire. La loi de diffusion en fonction du champ électrique est choisie de la forme :

$$
\frac{E D(E)}{v(E)}=\frac{k T}{q}
$$


avec une limite minimale $\left(D=8 \mathrm{~cm}^{2} \mathrm{~s}^{-1}\right)$ pour les champs électriques importants $[10,11]$.

Nous présentons figures 3 à 7 les résultats obtenus dans un fonctionnement en modulateur pour des tensions continues croissantes et inférieures à la tension d'avalanche. On peut remarquer (Fig. 3) pour les

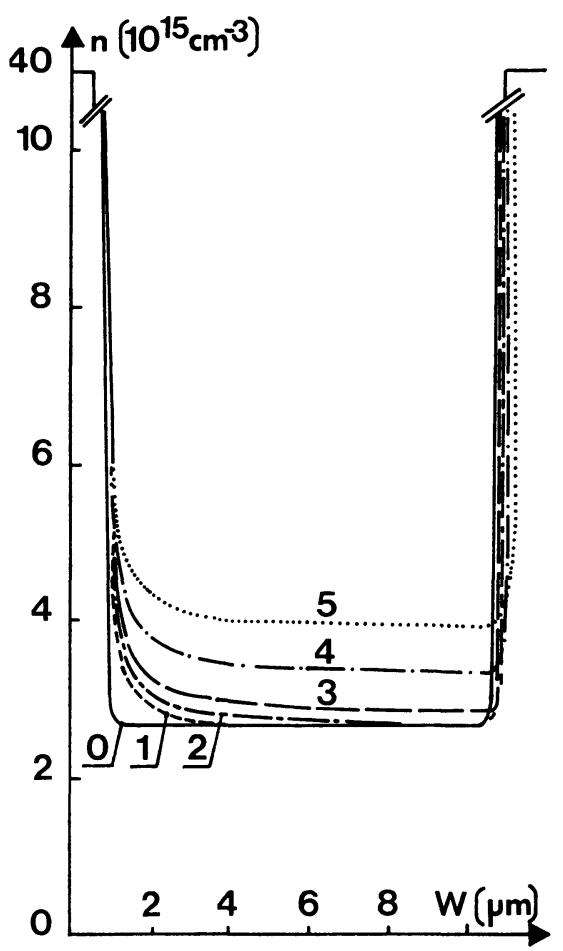

Fig. 3. - Profil de concentration des porteurs libres dans l'échantillon A pour différentes tensions de polarisation $V_{0} .0$. Profil de concentration en impuretés. $1 . V_{0}=14,7 \mathrm{~V}$. 2. $V_{0}=24,3 \mathrm{~V}$. 3. $V_{0}=39,1 \mathrm{~V}$. 4. $V_{0}=78,7 \mathrm{~V}$. 5. $V_{0}=125 \mathrm{~V}$.

[Free-carrier density distributions for some bias voltages in the device A. 0. Doping profile. $1 . V_{0}=14.7$ V. 2. $V_{0}=24.3 \mathrm{~V}$. 3. $V_{0}=39.1$ V. $4 . V_{0}=78.7$ V. $5 . V_{0}=125 \mathrm{~V}$.]

tensions les plus élevées le phénomène d'injection de porteurs dont la concentration devient largement supérieure à la concentration d'équilibre. En conséquence, le champ électrique dans la zone active est loin d'être uniforme (Fig. 4) et la caractéristique $I(V)$ ne présente pas de saturation en courant (Fig. 5). L'évolution de la conductance hyperfréquence en fonction de la tension de polarisation ou de la fréquence sont représentées figures 6 et 7 et l'on constate qu'il est possible pour des angles de transit voisins de $2 \dot{\pi}$ d'obtenir des valeurs de conductance largement inférieures à $g_{c}$. Les conclusions des calculs analytiques sont donc vérifiées et l'on peut d'ailleurs constater (Figs. 5 et 6 ) qu'un assez bon accord quantitatif

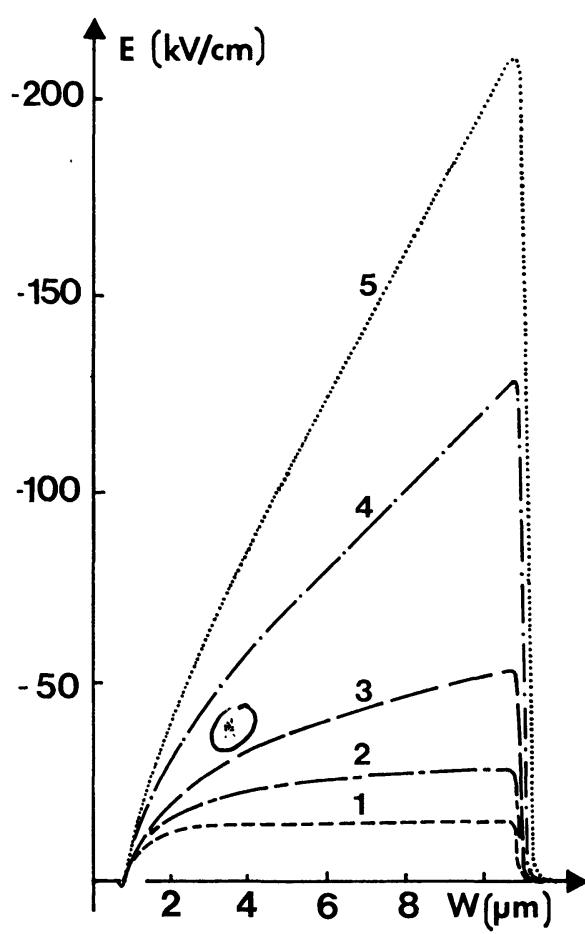

Fig. 4. - Carte du champ électrique pour différentes tensions de polarisation $V_{0}$ (échantillon A). 1. $V_{0}=14,7 \mathrm{~V}$. 2. $V_{0}=24,3 \mathrm{~V}$. 3. $V_{0}=39,1$ V. 4. $V_{0}=78,7$ V. 5 . $V_{0}=125 \mathrm{~V}$.

[Electric field distributions for some bias voltages. 1. $V_{0}=14.7 \mathrm{~V}$. 2. $V_{0}=24.3$ V. $3 . V_{0}=39.1$ V. $4 . V_{0}=78.7$ V. $5 . V_{0}=125$ V.]

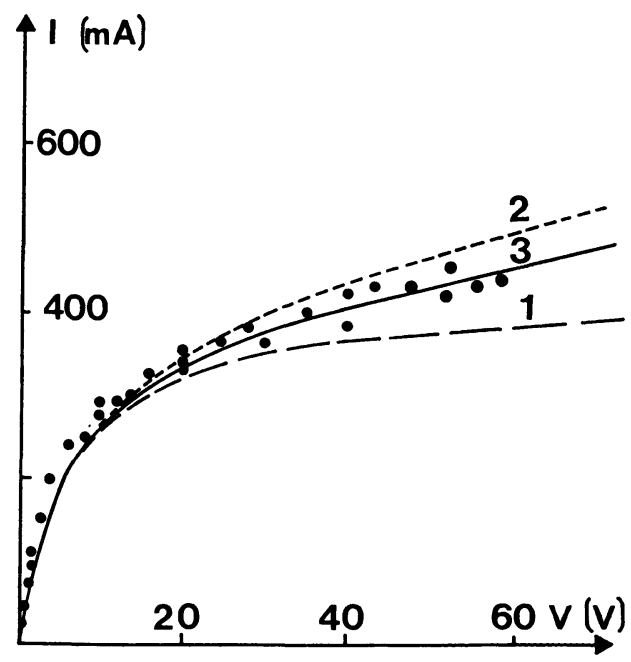

FIG. 5. - Caractéristiques courant-tension (échantillon A). 1. Effet lié à la limitation de vitesse : relation (3). 2. Effets liés à la limitation de vitesse et à la charge d'espace : relation (9). 3. Caractéristique $I(V)$ calculée numériquement. $\bullet$ Points expérimentaux pour trois échantillons différents.

$[I(V)$ characteristics (device A). 1 . With saturated velocity effect (equation 3). 2. With saturated velocity and space charge effects (equation 9). 3. Computed $I(V)$ characteristic. - Experimental results for three devices.] 
est obtenu entre la théorie analytique et les calculs numériques.

Les résultats obtenus pour un fonctionnement en limiteur (c'est-à-dire lorsque l'échantillon est soumis à

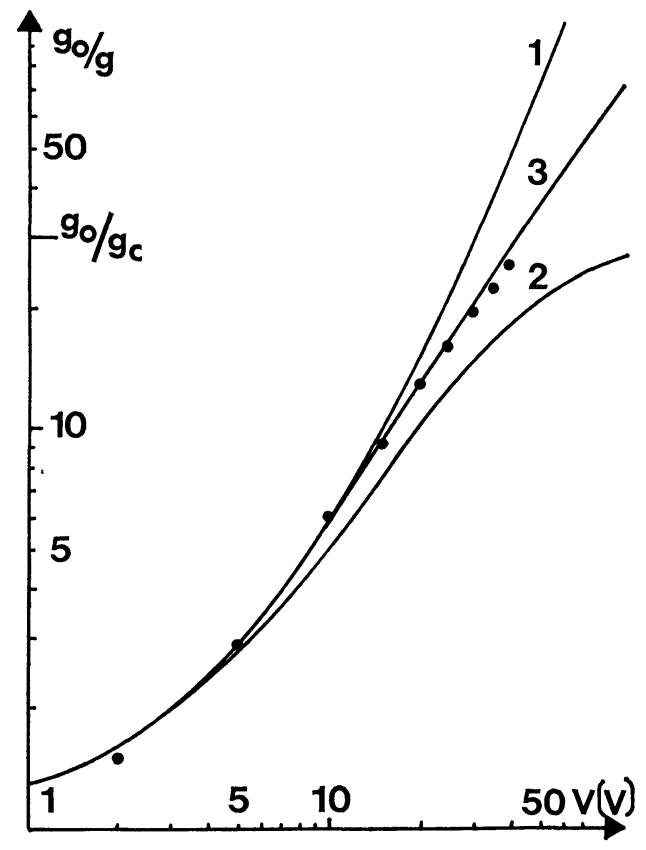

FIG. 6. - Variation de la conductance hyperfréquence $(F=10 \mathrm{GHz})$ en fonction de la tension de polarisation (échantillon A). 1. Effet lié à la limitation de la vitesse : relation (4). 2. Effets liés à la limitation de la vitesse et à la charge d'espace : relation (13). 3. Méthode numérique. • Points expérimentaux.

[Microwave conductance $(F=10 \mathrm{GHz})$ versus the bias voltage (device A). 1. With saturated velocity effect (equation 4). 2. With saturated velocity and space charge effects (equation 13). 3. Computed results. • Experimental results.]

une tension hyperfréquence croissante) sont donnés figures $8,9,10$. On remarque figure 8 que la conductance hyperfréquence ne peut être inférieure à $g_{c}$. Le phénomène d'injection périodique des porteurs est illustré figure 9. La répartition du champ électrique qui en résulte (Fig. 10) est très différente de celle correspondant au fonctionnement en modulateur. En particulier, comme le prévoit la loi de Poisson, l'amplitude du champ électrique reste constante dans la partie de la structure où les porteurs n'ont pas eu le temps d'être injectés, lorsque la période de la tension appliquée est suffisamment faible.

Il reste une caractéristique importante à évaluer : le temps de réponse de l'échantillon placé dans un circuit quelconque alimenté par un générateur dont la f.e.m. présente une variation temporelle arbitraire $E(t)$. Le procédé de calcul est alors légèrement modifié : le

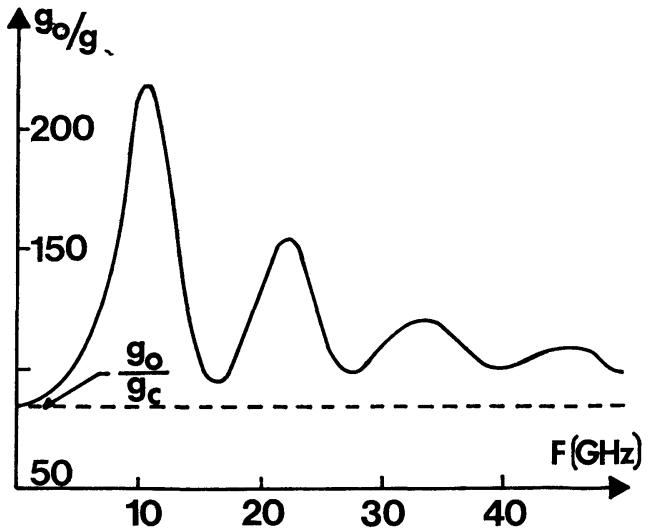

Fig. 7. - Variation en fonction de la fréquence de la conductance hyperfréquence pour une tension de polarisation maximale. Les caractéristiques de la structure sont les suivantes : $W=9,9 \mu$, $L=8,1 \mu, N_{\mathrm{D}}=10^{16} \mathrm{~cm}^{-3}$ (échantillon B).

[Microwave conductance versus frequency for a maximal bias voltage. The physical parameters of the device are : $W=9.9 \mu$, $L=8.1 \mu, N_{\mathrm{D}}=10^{16} \mathrm{~cm}^{-3}$ device B.]

courant traversant l'échantillon à l'instant $t_{0}$ étant fixé, on en déduit la tension aux bornes de l'échantillon $V\left(t_{\mathrm{o}}\right)$, l'application de la loi d'ohm connaissant les caractéristiques du circuit et la f.e.m. $E\left(t_{0}\right)$ permet alors le calcul du courant à l'instant ultérieur $I\left(t_{\mathrm{o}}+\Delta t\right)$ et le cycle de calcul recommence pour obtenir $V\left(t_{\mathrm{o}}+\Delta t\right)$ et $I\left(t_{\mathrm{o}}+2 \Delta t\right)$. On en déduit ainsi

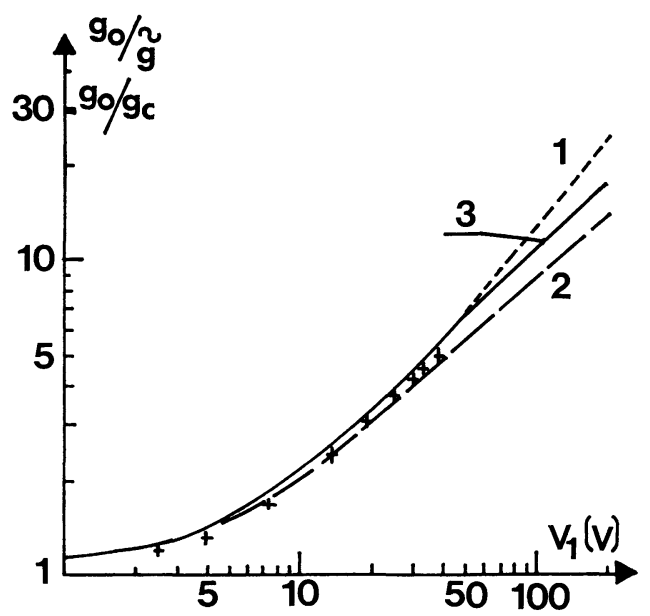

Fig. 8. - Variation de la conductance hyperfréquence $(F=9,375 \mathrm{GHz})$ en fonction de l'amplitude crête de la tension hyperfréquence (échantillon $\mathrm{A}): 1$, effet lié à la limitation de la vitesse : relation (5); 2 , effets liés à la limitation de la vitesse : relation (14) et à la charge d'espace; 3 , méthode numérique; $x$, points expérimentaux.

[Microwave conductance $(F=9.375 \mathrm{GHz})$ versus the peak value of the microwave bias voltage : 1 , with saturated velocity effect (equation 5); 2 , with saturated velocity and space charge effects (equation 14); 3 , computed results ; $\times$, experimental results.] 

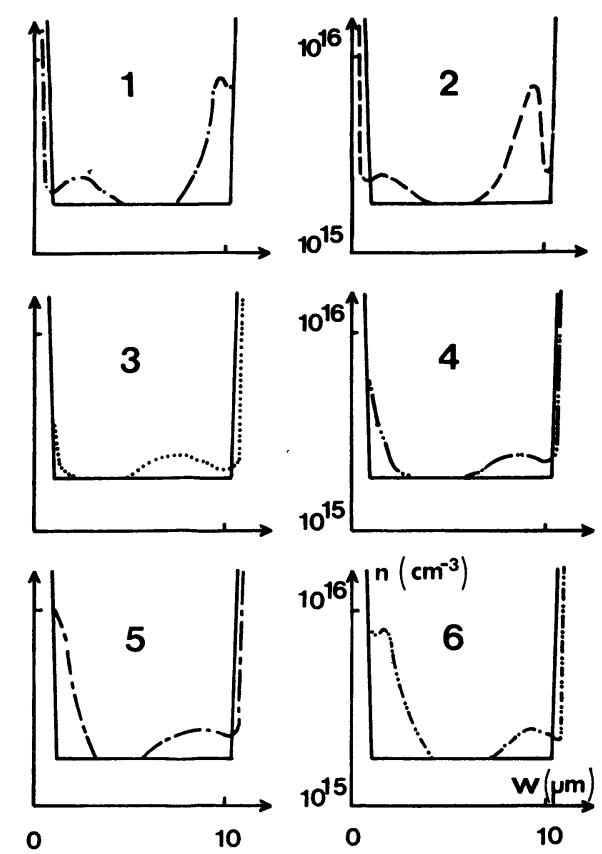

Fig. 9. - Profil de la concentration des porteurs (échantillon A) pour une tension de polarisation hyperfréquence $(F=10 \mathrm{GHz})$ :

$$
\begin{aligned}
& 1 t=0, \quad 2 t=\frac{T}{10}, \quad 3 t=\frac{2 T}{10}, \\
& 4 t=\frac{3 T}{10}, \quad 5 t=\frac{4 T}{10}, \quad 6 t=\frac{5 T}{10} .
\end{aligned}
$$

REMARQue. - Les profils aux instants $t$ et $t+T / 2$ sont symétriques par rapport à un axe passant par le milieu de la structure.

[Instantaneous distributions of the carrier density (device A) for a microwave bias voltage $(F=10 \mathrm{GHz})$ :

$$
\begin{array}{lll}
1 t=0 & 2 t=\frac{T}{10} & 3 t=\frac{2 T}{10} \\
4 t=\frac{3 T}{10} & 5 t=\frac{4 T}{10} & 6 t=\frac{5 T}{10}
\end{array}
$$

Note. - The middle axis of the structure is a symetrical axis for the instantaneous distributions at $t$ and $t+T / 2$.]

l'évolution temporelle du courant et de la tension aux bornes de l'échantillon. A titre d'exemple, nous pouvons simuler le fonctionnement d'un modulateur lorsque l'échantillon est soumis dans un circuit purement résistif à une tension de commande en échelon. Les résultats obtenus sont illustrés figures 11 et 12 ; on remarque figure 11 que l'application d'un échelon de tension important se traduit par l'injection d'un paquet d'électrons qui se propagent en s'étalant vers l'anode; le régime stationnaire n'est obtenu qu'après un temps qui est de l'ordre de grandeur du temps de transit. Ce phénomène se traduit par ailleurs sur les caractéristiques $I(t)$ (Fig. 12) où l'on remarque que le courant passe par un maximum au moment où le paquet d'électrons arrive à l'anode. Nous constatons sur cette figure une propriété intéressante de ce type de

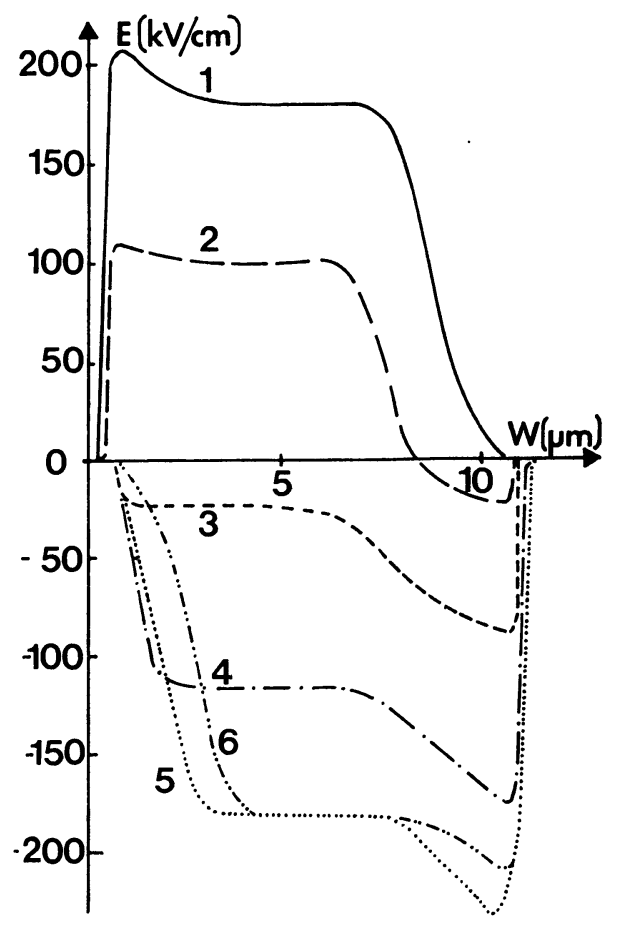

Fig. 10. - Carte du champ électrique dans la structure (échantillon A) pour une tension de polarisation hyperfréquence $(F=10 \mathrm{GHz}) .1 t=0 \mathrm{~V}(t)=-156 \mathrm{~V} ; 2 t=T / 10 V(t)=-66,5 \mathrm{~V}$; $3 t=2 T / 10 V(t)=43,1 \mathrm{~V} ; 4 t=3 T / 10 V(t)=127 \mathrm{~V}$; $5 t=4 T / 10 V(t)=176 \mathrm{~V} ; 6 t=5 T / 10 V(t)=156 \mathrm{~V}$.

[Instantaneous distributions of the field in the structure (device A) for a microwave bias voltage $(F=10 \mathrm{GHz}) .1 t=0 \mathrm{~V}(t)=-156 \mathrm{~V}$; $2 t=T / 10 V(t)=-66.5 \mathrm{~V} ; 3 t=2 T / 10 V(t)=43.1 \mathrm{~V}$; $4 t=3 T / 10 V(t)=127 \mathrm{~V} ; \quad 5 t=4 T / 10 V(t)=176 \mathrm{~V}$; $6 t=5 T / 10 V(t)=156 \mathrm{~V}$.]

composant : les effets de limitation ou de modulation aux temps courts sont plus importants qu'en régime stationnaire puisque le phénomène d'injection n'a pas encore eu le temps d'intervenir.

En conclusion, les études théoriques et numériques précédentes ont montré que l'admittance hyperfréquence d'une structure semiconductrice unipolaire soumise à une tension électrique (continue ou hyperfréquence) importante est caractérisée par une conductance qui peut varier largement en fonction de l'amplitude de la tension appliquée alors que la capacité tenant compte du courant de déplacement est pratiquement constante. Par ailleurs, l'étude du régime transitoire a montré que les variations de conductance obtenues suivent très rapidement l'application du signal de polarisation. Nous allons dans le chapitre suivant donner quelques vérifications expérimentales de ces constatations.

2. Vérifications expérimentales. - Afin de vérifier expérimentalement l'étude théorique précédente, des échantillons $\mathrm{N}^{+} \mathrm{N} \mathrm{N}^{+}$au silicium ont été réalisés 


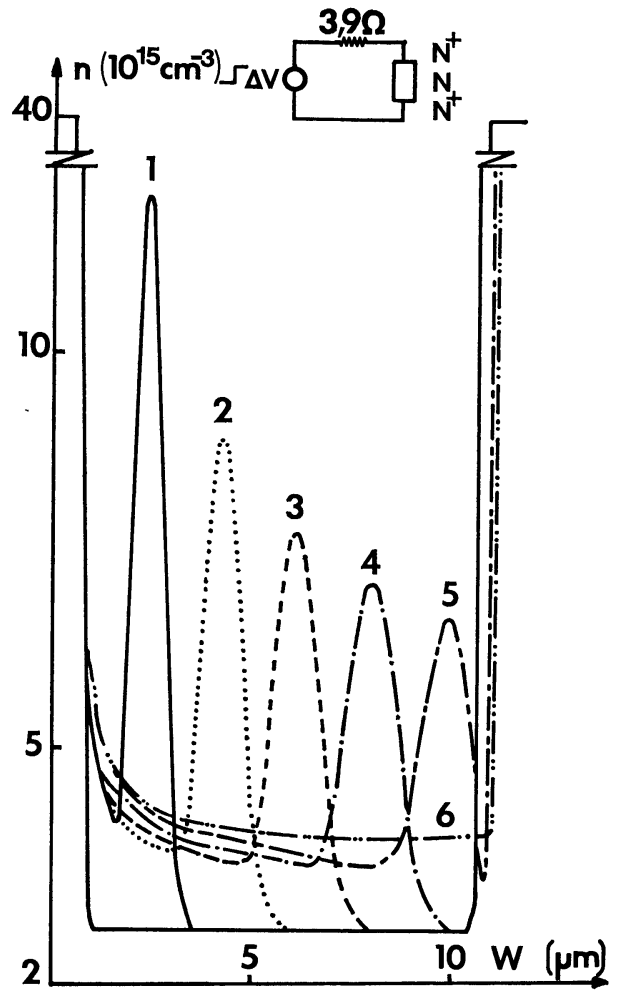

FIG. 11. - Profil de concentration des porteurs libres (échantillon A) à différents instants après l'application d'un échelon de tension $\Delta V=127 \mathrm{~V}$ à l'instant initial : $1 t=20 \mathrm{ps} ; 2 t=40 \mathrm{ps}$;

$$
3 t=60 \mathrm{ps} ; 4 t=80 \mathrm{ps} ; 5 t=100 \mathrm{ps} ; 6 t=200 \mathrm{ps} \text {. }
$$

REMARQUE. - Nous utilisons ici une résistance interne du générateur très faible pour amplifier le phénomène d'injection de porteurs.

[Instantaneous distributions of carriers (device A) following the voltage step $\Delta V=127 \mathrm{~V} ; 1 t=20 \mathrm{ps} ; 2 t=40 \mathrm{ps} ; 3 t=60 \mathrm{ps}$; $4 t=80 \mathrm{ps} ; 5 t=100 \mathrm{ps} ; 6 t=200 \mathrm{ps}$.]

NotE. - A low generator resistance is used in order to increase the carrier injection effect.

en collaboration avec la société L.T.T. Les difficultés de réalisation sont dues d'une part, au fonctionnement en champs électriques élevés (nécessité d'éviter les claquages superficiels) et d'autre part à l'élaboration de bons contacts ohmiques supportant des densités de courant importantes au voisinage de champs électriques élevés.

Les résultats cités dans cet article concernent uniquement la première série d'échantillons réalisés dont les caractéristiques générales de la zone active sont les suivantes : silicium de résistivité $1,6 \Omega \mathrm{cm}$, longueur 10 microns, surface $1 \times 10^{-4} \mathrm{~cm}^{2}$. Ces échantillons de type mésa sont élaborés à partir d'une épitaxie $\mathrm{N} \mathrm{N}^{+}$, d'une diffusion $\mathrm{N}^{+}$suivie d'une métallisation multiface. La technologie utilisée n'est pas encore optimalisée et les tensions de claquage observées sont un peu plus faibles que les tensions d'ava-

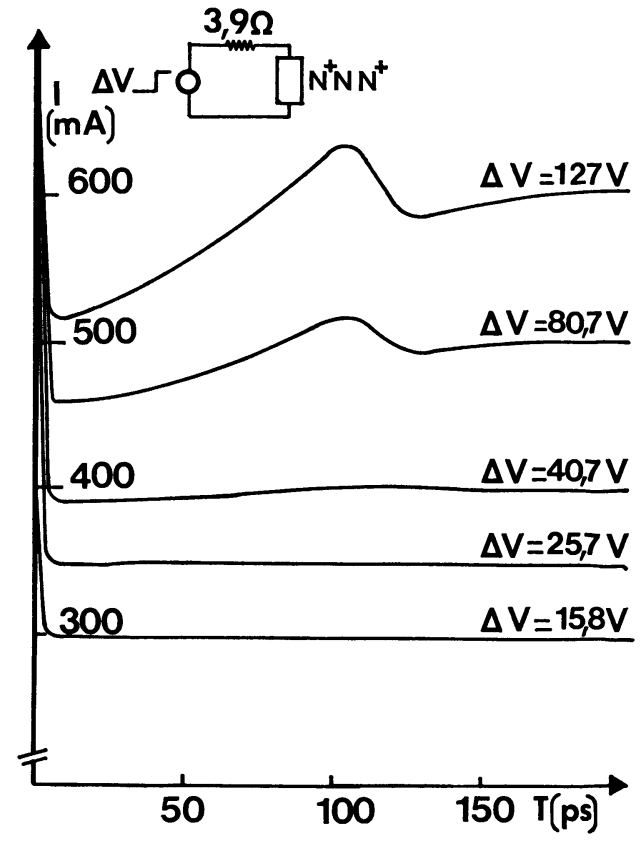

FIG. 12. - Evolution du courant traversant le composant en fonction du temps quand le générateur délivre l'échelon de tension, $\Delta V$ à l'instant initial.

[Current versus the time for a voltage step $\Delta V$.]

lanche théoriques. Les caractéristiques expérimentales $I=f(V)$ (fig. 5) ont été tout d'abord déterminées avec des impulsions de tension de 200 nanosecondes de durée et de fréquence de répétition de $25 \mathrm{~Hz}$. De cette manière, on évite tout échauffement moyen et instantané de la zone active de la structure. Les résultats expérimentaux reportés correspondent à trois échantillons différents et sont très voisins de ceux calculés numériquement. Les mesures de l'impédance hyperfréquence effectuées à $10 \mathrm{GHz}$ pendant la durée d'application de la tension de commande sont réalisées sur des échantillons montés en boitiers hyperfréquences classiques ou directement incorporés dans un circuit microstrip. Les variations du rapport $g_{\mathrm{o}} / g$ en fonction de l'amplitude de la tension de polarisation sont reportés figure 6 . On constate un bon accord avec les rèsultats de l'étude numérique. Des mesures d'impédance ont ensuite été effectuées à $9,375 \mathrm{GHz}$, avec des impulsions hyperfréquences de 1,75 microseconde de durée et une fréquence de répétition de $60 \mathrm{~Hz}$. Les variations expérimentales du rapport $g_{\mathrm{o}} / \tilde{g}$ sont représentées figure 8 en fonction de l'amplitude de l'onde hyperfréquence; on remarque ici encore que la théorie effectuée donne des résultats conformes à l'expérience.

En résumé, les premières mesures expérimentales effectuées ont démontré l'exactitude des phénomènes prévus théoriquement, il reste maintenant à envisager l'utilisation pratique des structures $\mathrm{N}^{+} \mathrm{N} \mathrm{N}^{+}$pour la 
réalisation de modulateurs et limiteurs hyperfréquences ultra-rapides.

3. Applications hyperfréquences. - 3. 1. ÉvaluATIONS THÉORIQUES DES PERFORMANCES. - Un grand nombre de circuits hyperfréquences peuvent être utilisés pour réaliser un dispositif modulateur ou limiteur.
Le plus souvent, l'échantillon semiconducteur est placé en série dans la ligne de transmission reliant le générateur d'impédance interne $R_{\mathrm{L}}$ et le récepteur dont l'impédance est supposée résistive et adaptée : c'est le montage série. Un accord uniquement réactif est placé en parallèle sur l'échantillon et l'atténuation obtenue (exprimée en $\mathrm{dB}$ ) s'exprime par la relation :

$$
\alpha(\mathrm{dB})=10 \log \left\{\left[1+\frac{g(V) G_{\mathrm{L}}}{2\left(g^{2}(V)+B_{\mathrm{T}}^{2}\right)}\right]^{2}+\left[\frac{B_{\mathrm{T}} G_{\mathrm{L}}}{2\left(g^{2}(V)+B_{\mathrm{T}}^{2}\right)}\right]^{2}\right\}
$$

où $B_{\mathrm{T}}=B+B_{\mathrm{A}}$ : susceptance du semiconducteur + susceptance de l'accord $G_{\mathrm{L}}$ : conductance du générateur et du récepteur,

$g(V)$ : conductance du semiconducteur fonction de la tension de polarisation.

En faisant varier la susceptance de l'accord on peut annuler le terme réactif et dans ce cas la relation (20) devient :

$$
\alpha(\mathrm{dB})=20 \log \left[1+\frac{G_{\mathrm{L}}}{2 g(V)}\right]
$$

Dans le montage type parallèle, le composant semiconducteur et son dispositif d'accord sont placés en parallèle sur la ligne de transmission. L'expression de l'atténuation devient :

$$
\alpha(\mathrm{dB})=10 \log \left[\left(1+\frac{g(V)}{2 G_{\mathrm{L}}}\right)^{2}+\left(\frac{B_{\mathrm{T}}}{2 G_{\mathrm{L}}}\right)^{2}\right]
$$

et en réalisant l'accord on obtient :

$$
\alpha(\mathrm{dB})=20 \log \left[1+\frac{g(V)}{2 G_{\mathrm{L}}}\right]
$$

Dans le fonctionnement en modulateur (montage série ou parallèle), on modifie la conductance $g(V)$ en faisant varier la tension de polarisation appliquée à l'échantillon. En désignant par $g_{o}$ et $g$ les valeurs maximale et minimale présentées par la conductance, on en déduit facilement la relation donnant les atténuations maximale et minimale présentées par le modulateur soit :

$\alpha_{\text {MAX }}(\mathrm{dB})=20 \log \left[1+\frac{g_{0}}{g}\left(10^{\frac{\alpha_{\min }(\mathrm{dB})}{20}}-1\right)\right]$

Cette relation est encore valable dans le fonctionnement en limiteur, mais c'est la variation de la tension hyperfréquence aux bornes de l'échantillon qui entraîne les modifications de la conductance présentée par l'échantillon.

Ainsi, l'obtention d'atténuations maximales élevées associées à des pertes d'insertion faibles nécessite l'emploi d'un échantillon de semiconducteur présen- tant un rapport $g_{0} / g$ le plus grand possible. Cette constatation est illustrée figure 13 où à partir de la relation (24) nous avons représenté en fonction du rapport $g_{0} / g$ l'atténuation maximale obtenue pour différentes valeurs des pertes d'insertion. A partir de la figure 13 et des valeurs $g_{0} / g$ données dans les tableaux I et II, il est possible de prévoir les performances susceptibles d'être atteintes par des modulateurs et des limiteurs réalisés avec des structures semiconductrices $\mathrm{N}^{+} \mathrm{N} \mathrm{N}^{+}$en $\mathrm{Ge}, \mathrm{Si}, \mathrm{GaAs}$, $\mathrm{Ga}_{x} \mathrm{In}_{1-x}, \mathrm{Sb}$.

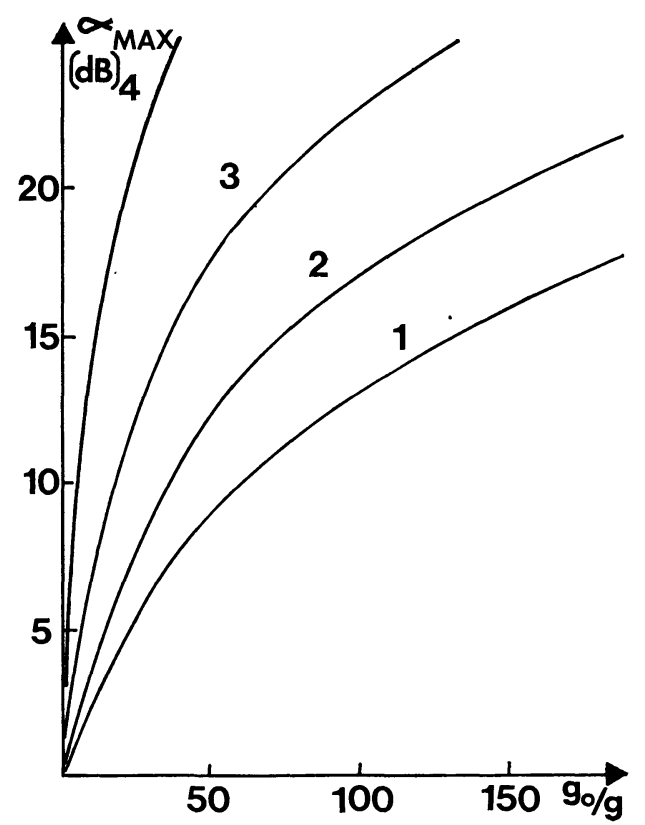

FIG. 13. - Montage en transmission. Atténuation maximale obtenue pour différentes valeurs des pertes d'insertion en fonction du rapport $g_{0} / g$ ( $g_{0}$ est la conductance présentée par l'échantillon sous une faible tension de polarisation, $g$ est la conductance pour une tension de polarisation maximale) : $1 \alpha_{\min }=0,3 \mathrm{~dB}$;

$$
2 \alpha_{\min }=0,5 \mathrm{~dB} ; 3 \alpha_{\min }=1 \mathrm{~dB} ; 4 \alpha_{\min }=3 \mathrm{~dB} \text {. }
$$

[Transmission configuration. Maximal attenuation for some insertion loss values versus the ratio $g_{0} / g\left(g_{0}\right.$ : low bias voltage conductance, $g$ maximal bias voltage conductance) : $1 \alpha_{\min }=0.3 \mathrm{~dB}$; $2 \alpha_{\min }=0.5 \mathrm{~dB} ; 3 \alpha_{\min }=1 \mathrm{~dB} ; 4 \alpha_{\min }=3 \mathrm{~dB}$.] 
On peut cependant remarquer que des performances supérieures peuvent être obtenues à l'aide d'un montage en réflexion mais un circulateur doit alors être utilisé. Dans ce cas, l'atténuation maximale est alors pratiquement infinie (si le circulateur est parfait) lorsque la conductance de l'échantillon est égale à la conductance caractéristique de la ligne de transmission utilisée et l'atténuation minimale est donnée par la relation :

$$
\alpha_{\min }(\mathrm{dB})=20 \log \left|\frac{G_{\mathrm{L}}+g_{0}}{G_{\mathrm{L}}-g_{0}}\right|
$$

Illustrons maintenant ces considérations par quelques exemples plus précis des performances que peuvent présenter des modulateurs et des limiteurs réalisés selon ces principes. Les figures 14 et 15 concernent un modulateur utilisant un composant en silicium et l'on peut remarquer que des atténuations importantes peuvent être obtenues dans des bandes passantes élevées. Nous étudions figures 16 et 17 le fonctionnement en limiteur : les variations calculées numériquement de la puissance de sortie en fonction de la puissance d'entrée sont reportées figure 16 pour différentes structures en silicium ou en arséniure de gallium. On peut remarquer l'amélioration des performances obtenues grâce à l'emploi d'un semiconducteur de grande mobilité et de faible champ critique. Le comportement impulsionnel théorique d'un limiteur silicium est enfin illustré figure 17; on note que l'effet de limitation est pratiquement instantané et que le spike est négligeable.

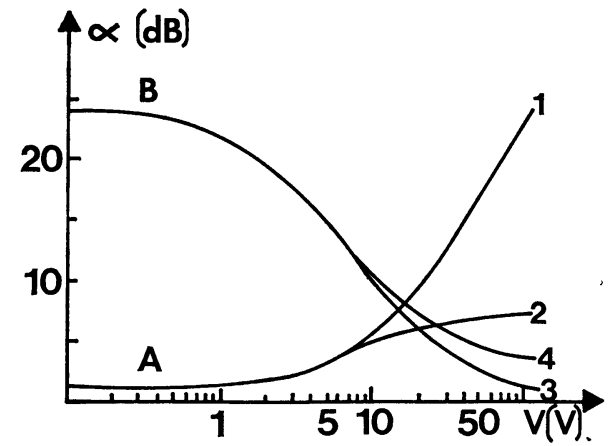

Fig. 14. - Fonctionnement en modulateur à $10 \mathrm{GHz}$. Caractéristiques de la structure semiconductrice : $N_{\mathrm{D}}=4 \times 10^{15} \mathrm{~cm}^{3}$, $W=9.9 \mu, L \simeq 8,1 \mu, S=10^{-4} \mathrm{~cm}^{2}$. A) Montage série, impédance de la ligne $Z_{\mathrm{L}}=32 \Omega: 1$ ) avec accord parallèle; 2) sans accord parallèle. B) Montage parallèle, impédance de la ligne $Z_{\mathrm{L}}=234 \Omega: 3$ ) avec accord parallèle ; 4) sans accord parallèle.

[Modulator operating $F=10 \mathrm{GHz}$; the structure parameters are : $N_{\mathrm{D}}=4 \times 10^{15} \mathrm{~cm}^{-3}, W=9.9 \mu, L=8.1 \mu, S=10^{-4} \times \mathrm{cm}^{2}$. A) Serial configuration, characteristic impedance $Z_{\mathrm{L}}=32 \Omega$ : 1) with shunt tuning;2) without shunt tuning. B) Shunt configuration, characteristic impedance $Z_{\mathrm{L}}=234 \Omega: 3$ ) with shunt tuning ; 4) without shunt tuning.]

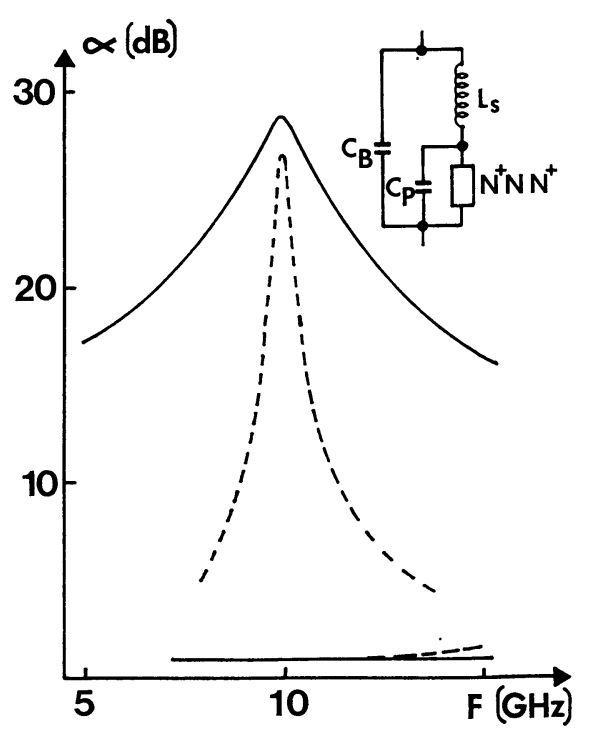

Fig. 15. - Evolution en fonction de la fréquence des atténuations maximales $\left(V_{0}=130 \mathrm{~V}\right)$ et minimale $\left(V_{0}=0\right)$ d'un modulateur série à accord parallèle pour $F=10 \mathrm{GHz}$. Impédance de la ligne $Z_{\mathrm{L}}=45 \Omega$ : - sans encapsulation; -..-- avec encapsulation $\left(L_{\mathrm{s}}=0,4 \mathrm{nH}, C_{\mathrm{B}}=0,2 \mathrm{pF}, C_{\mathrm{P}}=0,04 \mathrm{pF}:\right.$ valeurs typiques d'un boîtier hyperfréquence classique).

[Maximal attenuations $\left(V_{0}=130 \mathrm{~V}\right)$ and insertion losses $\left(V_{0}=0\right)$ for a shunt tuning $(F=10 \mathrm{GHz})$ serial modulator versus the frequency characteristic impedance $Z_{\mathrm{L}}=45 \Omega:-$ without package ; --.-- with standard package $\left(L_{\mathrm{s}}=0.4 \mathrm{nH}, C_{\mathrm{B}}=0.2 \mathrm{pF}\right.$, $\left.C_{\mathrm{P}}=0.04 \mathrm{pF}\right)$.]

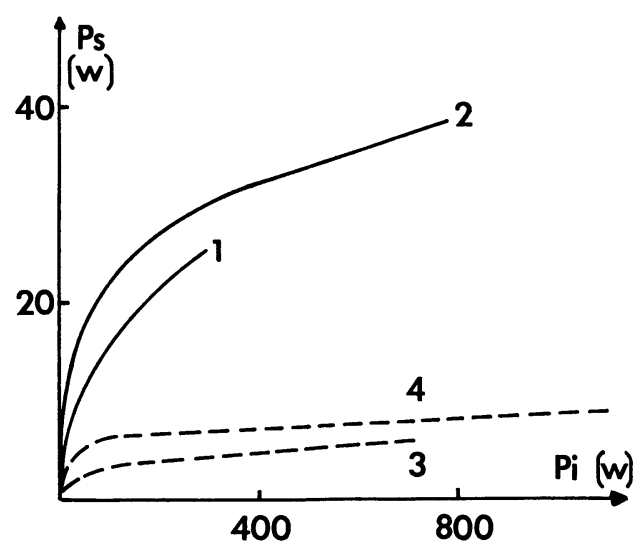

Fig. 16. - Puissance de sortie en fonction de la puissance d'entrée pour un limiteur série à accord parallèle : $F=10 \mathrm{GHz}, \alpha_{\min }=1 \mathrm{~dB}$, 1 silicium $N_{\mathrm{D}}=4 \times 10^{15} \mathrm{~cm}^{-3}, \alpha_{\max }=10,6 \mathrm{~dB} ; 2$ silicium $N_{\mathrm{D}}=1 \times 10^{16} \mathrm{~cm}^{-3}, \alpha_{\max }=13,1 \mathrm{~dB} ; 3$ arséniure de gallium $N_{\mathrm{D}}=4 \times 10^{15} \mathrm{~cm}^{-3}, \alpha_{\max }=20,4 \mathrm{~dB} ; 4$ arséniure de gallium $N_{\mathrm{D}}=10^{16} \mathrm{~cm}^{-3}, \alpha_{\max }=23,4 \mathrm{~dB}$.

[Output versus input powers for a shunt tuning serial limiter $F=10 \mathrm{GHz}, \alpha_{\min }=1 \mathrm{~dB} ; 1$ silicon $N_{\mathrm{D}}=4 \times 10^{15} \mathrm{~cm}^{-3}$, $\alpha_{\max }=10.6 \mathrm{~dB} ; 2$ silicon $N_{\mathrm{D}}=1 \times 10^{16} \mathrm{~cm}^{-3}, \alpha_{\max }=13.1 \mathrm{~dB}$; 3 gallium arsenide $N_{\mathrm{D}}=4 \times 10^{15} \mathrm{~cm}^{-3}, \alpha_{\max }=20.4 \mathrm{~dB} ; 4$ gallium arsenide $N_{\mathrm{D}}=10^{16} \mathrm{~cm}^{-3}, \alpha_{\max }=23.4 \mathrm{~dB}$.]

3. 2. Premiers résultats eXpérimentauX. - En vue de fournir une illustration expérimentale de cette 


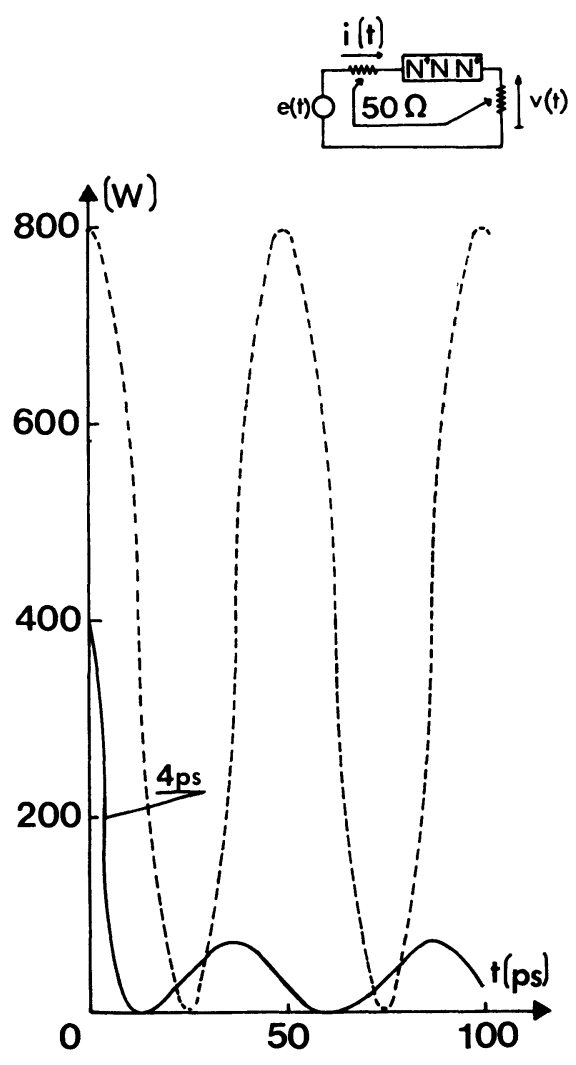

Fig. 17. - Fonctionnement en limiteur : $F=10 \mathrm{GHz}, \alpha_{\min }=1 \mathrm{~dB}$; évolution de $e(t) . i(t)$ en fonction du temps $\bar{P}_{e}=400 \mathrm{~W}$; évolution de $v(t) . i(t)$ en fonction du temps $\dot{P}_{\mathrm{s}}^{\mathrm{e}}=32,3 \mathrm{~W}$.

[Limiter operating : $\left.F=10 \mathrm{GHz}, \alpha_{\min }=1 \mathrm{~dB} ;-----\right) e(t) . i(t)$ time dependence $\bar{P}_{\mathrm{e}}=400 \mathrm{~W} ;-v(t) . i(t)$ time dependence $\left.\bar{P}_{\mathrm{s}}=32.3 \mathrm{~W}.\right]$

étude, nous présentons quelques résultats préliminaires obtenus avec des modulateurs et des limiteurs à commutation de mobilité. Les maquettes réalisées sont susceptibles d'améliorations et les résultats définitifs obtenus en collaboration avec la société L.T.T. seront publiés ultérieurement. Deux types de structures ont été réalisés en guide d'onde : un montage en transmission et un montage en réflexion associé à un circulateur. Nous donnons figures 18 et 19 l'évolution des atténuations obtenues en fonction de la tension de commande (modulateur) et de la puissance incidente (limiteur). Les deux dernières figures illustrent la propriété la plus intéressante de ce type de composant c'est-à-dire leur rapidité. Il apparaît (figure 20) que le temps de réponse du modulateur n'est pas mesurable, son évaluation nécessiterait un signal de commande de temps de montée beaucoup plus faible (inférieur à $50 \mathrm{ps}$ ). La réponse temporelle du limiteur est étudiée figure 21 ; une impulsion hyperfréquence incidente présentant déjà un spike important (c'est le cas habituellement à la sortie des limiteurs haute puissance) est appliquée à son entrée, on voit que le spike à la sortie a pratiquement disparu.

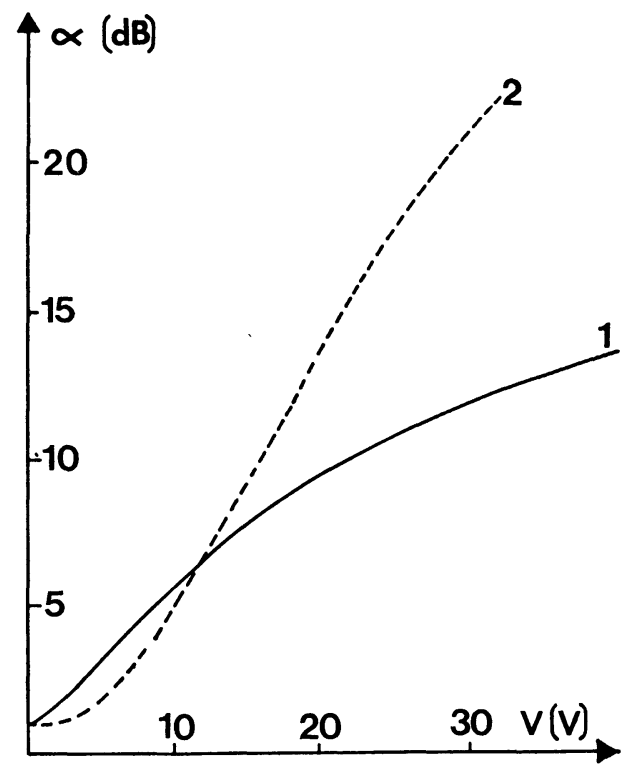

Fig. 18. - Fonctionnement en modulateur à $10 \mathrm{GHz}$. Atténuation mesurée en fonction de la tension de polarisation appliquée à l'échantillon : 1 . montage en transmission ; 2 . montage en réflexion.

[Modulator operating at $10 \mathrm{GHz}$. Experimental attenuations versus the bias voltage : 1 . transmission configuration; 2 . reflection configuration.]

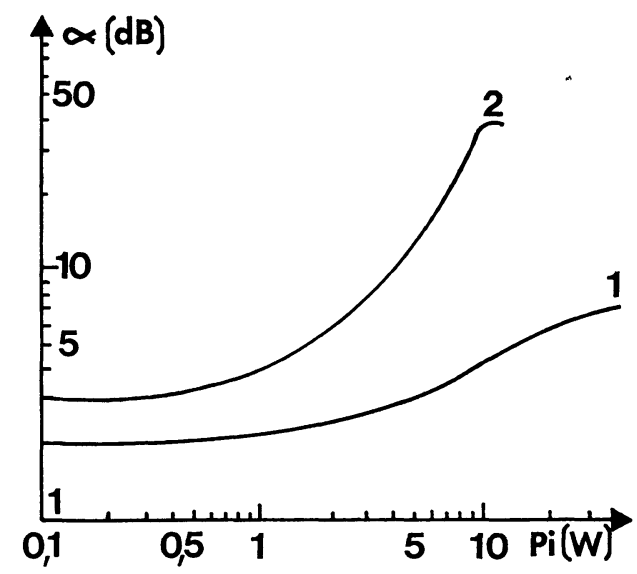

FIG. 19. - Fonctionnement en limiteur à $9,375 \mathrm{GHz}$. Atténuation mesurée en fonction de la puissance hyperfréquence incidente (échantillon A) : 1. montage en transmission; 2 . montage en réflexion.

[Limiter operating at $9.375 \mathrm{GHz}$. Experimental attenuations versus the input microwave power (device A) : 1 . transmission configuration ; 2. reflection configuration.]

4. Conclusion. - Dans ce travail, nous nous sommes efforcés d'étudier les possibilités d'un nouveau type de composant semiconducteur hyperfréquence. Nous avons montré que l'utilisation de la 


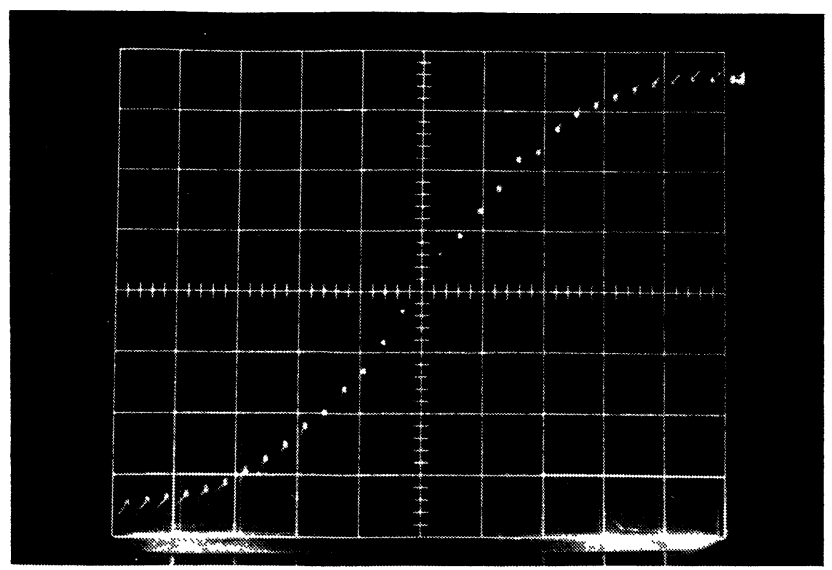

a)

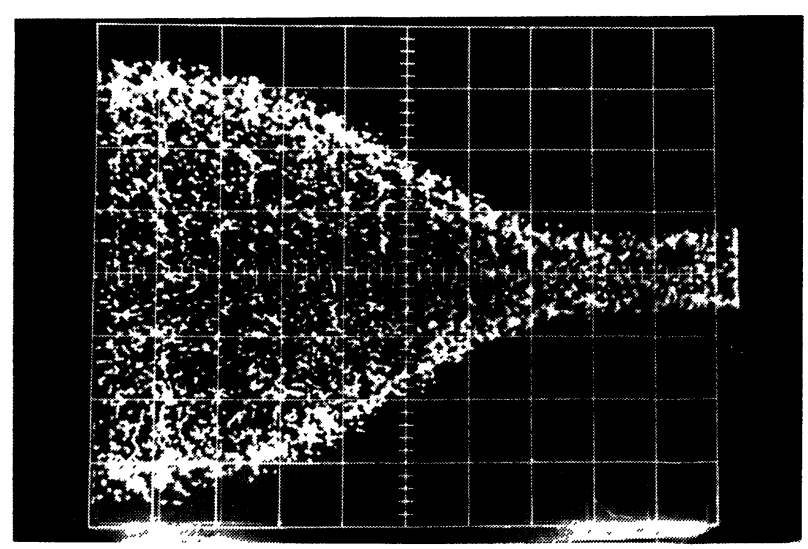

b)

FIG. 20. - Fonctionnement en modulateur à $10 \mathrm{GHz}$ (échantillon $\mathrm{A}): 1$. tension de polarisation appliquée à l'échantillon axe horizontal $1 \mathrm{div}=50 \mathrm{ps} ; 2$. onde hyperfréquence à la sortie du modulateur axe horizontal $1 \mathrm{div}=50 \mathrm{ps}$.

[Modulator operating at $10 \mathrm{GHz}$ (device A) : 1 . input bias voltage risetime (unit time $50 \mathrm{ps} / \mathrm{cm}$ ) ; 2 . output microwave response (unit time $50 \mathrm{ps} / \mathrm{cm})$.]

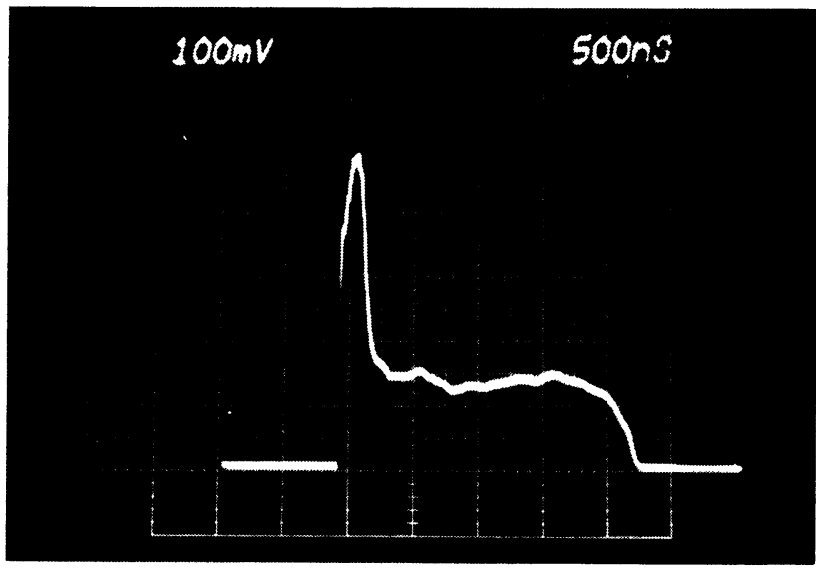

a)

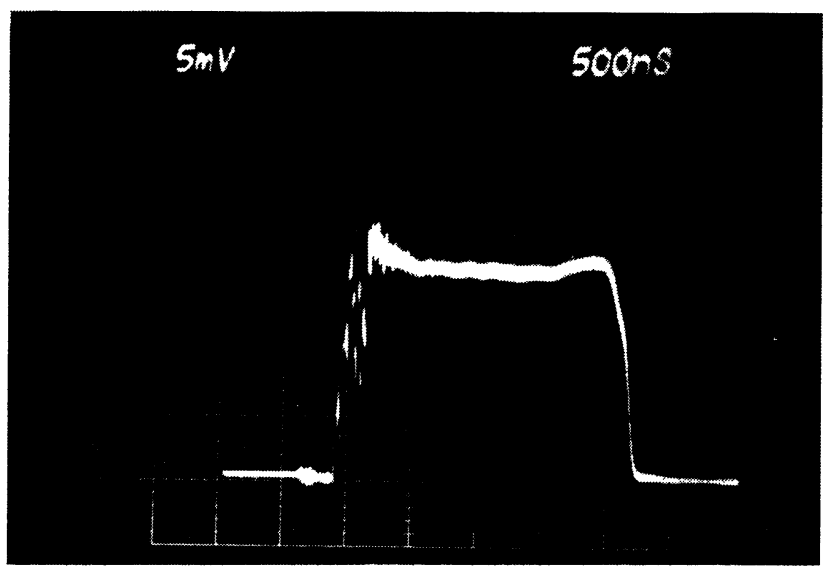

b)

FIG. 21. - Fonctionnement en limiteur $F=9,375 \mathrm{GHz}$ (échantillon A) : 1 . impulsion hyperfréquence incidente : $P_{\text {crête }}=30 \mathrm{~W}$, $P_{\text {plateau }}=3 \mathrm{~W} ; 2$. impulsion transmise par le limiteur $\alpha_{\text {crête }}=20 \mathrm{~dB}, \alpha_{\text {plateau }}=7 \mathrm{~dB}, \alpha_{\min }=3 \mathrm{~dB}$.

[Limiter operating $F=9.375 \mathrm{GHz}$ (device A) : $\bullet$ input microwave signal spike leakage $30 \mathrm{~W}$, flat leakage $3 \mathrm{~W}$; $\bullet$ limiter output signal spike leakage attenuation $=20 \mathrm{~dB}$, flat leakage attenuation $=7 \mathrm{~dB}$, insertion loss $=3 \mathrm{~dB}$.]

variation de mobilité des porteurs par effet de champ permet d'obtenir une modulation ultra-rapide ou une limitation quasi-instantanée d'une onde hyperfréquence.

Les quelques études expérimentales effectuées ont permis de vérifier la validité des prévisions théoriques. Les premiers résultats obtenus sur des maquettes non optimalisées réalisées à partir d'un composant en silicium (semiconducteur sans doute le moins adapté à ce type d'utilisation) montrent l'intérêt que pourraient présenter ces nouveaux dispositifs modulateurs et limiteurs hyperfréquences.

Remerciements. - Ce travail a été effectué avec le soutien de la D.R.M.E. Nous remercions par ailleurs Messieurs B. Chiron, G. Forterre, J. Marcoux et J. L. Assemat qui nous ont fourni quelques-uns des échantillons utilisés et avec lesquels nous avons eu de fructueuses discussions. Nous tenons enfin à exprimer nos remerciements à $\mathrm{J}$. Vanbremeersch pour toutes ses réalisations technologiques. 


\section{Bibliographie}

[1] Foggiato G. A. et Pearson G. L., Proc. IEEE, 60 (1972), 456.

[2] Immorlica A. et Pearson G., IEEE Trans. Electron Devices. ED 22 (1975), 829.

[3] Kallback B., Electron. Lett. 8 (1972), 476.

[4] Constant E. et Carnez B., Onde Electr. 56 (1976), 349.

[5] Hawashima M., Ohta K. et Kataoka S., Contribution to 1976 Symposium on GaAs and Related Compounds.

[6] Sakai K., Ixoma T., Adachi Y. et Yanai H., Electron. Lett. 10 (1974), 402.

[7] DasCalu D., Transit time effects in unipolar solid-states devices, Chap. XII (Abacus Press).
[8] MirCEA A. Contribution à l'étude de l'instabilité à fort signal et du bruit dans les dispositifs à transfert d'électron. Chap. 2 Thèse, Paris, 1976.

[9] WACHSPRESS E. L. Mathematical methods for digital computers. A. Ralston et HS. Wilf éditeur (John Wiley), 1967, ch. 10.

[10] Jacoboni C., Canali C., Ottaviani G. et Alberighi QuaRANTA A. Solid-State Electron 20 (1977) 77.

[11] Zimmermann J., Bonfils S., Leroy Y. et Constant E. Appl. Phys. Lett. 30 (1977). 